\title{
Mohs Micrographic Surgery During the COVID-19 Pandemic: Considering the Patient Perspective
}

\author{
Catherine N.Tchanque-Fossuo, MD, MS; Sabah Osmani, BA; Joseph M. Yardman-Frank, MPH; \\ Naiara S. Barbosa, MD
}

\section{PRACTICE POINTS}

- There is little evidence that supports a high risk for SARS-CoV-2 transmission during Mohs micrographic surgery when proper personal protective equipment and strict infection control protocols are in place.

- The effects of treatment delays due to COVID-19 on the patient experience have not been well studied, but the limited literature suggests a negative association.

- Shared decision-making and evaluation of individual patient risk factors and concerns should be considered when deciding whether to postpone skin cancer treatment.

\section{Guidelines on Skin Cancer Surgeries During the COVID-19 Pandemic}

At the start of the COVID-19 pandemic, the Centers for Disease Control and Prevention issued recommendations to decrease the spread of SARS-CoV-2 and optimize the use of personal protective equipment (PPE) for frontline workers. ${ }^{1}$ In the field of dermatologic surgery, the American College of Mohs Surgery, the National Comprehensive Cancer Network, the American Society for Dermatologic Surgery, and the American Academy of Dermatology made recommendations to postpone nonessential and nonurgent procedures. ${ }^{2-4}$ The initial guidelines of the American College of Mohs Surgery advised cancellation of all elective surgeries and deferred treatment of most cases of basal cell carcinoma for as long as 3 months; low-risk squamous cell carcinoma (SCC) and melanoma in situ treatment was deferred for as long as 2 or 3 months. ${ }^{3}$ Additional recommendations were made to reserve inpatient visits for suspicious lesions and high-risk cancers, postpone other nonessential and nonurgent appointments, and utilize telemedicine whenever possible. ${ }^{5}$

These recommendations led to great uncertainty and stress for patients and providers. Although numerous important variables, such as patient risk factors, severity of disease, availability of PPE and staff, and patient-toprovider transmission were considered when creating these guidelines, the patient's experience likely was not a contributing factor.

\section{COVID-19 Transmission During Mohs Surgery}

There have been concerns that surgeons performing Mohs micrographic surgery (MMS) might be at an increased risk for COVID-19, given their close contact with high-risk sites (ie, nose, mouth) and cautery-generated aerosols; most of the estimated transmission risk associated with MMS has been based on head and neck surgery experience and publications. ${ }^{6-8}$ Tee and colleagues ${ }^{9}$ recently published their institution's MMS COVID-19 preventive measures, which, to their knowledge, have prevented all intraoperative transmission of SARS-CoV-2, even in disease-positive patients. Currently, evidence is lacking to support a high risk for SARS-CoV-2 transmission during MMS when proper PPE and personal hygiene measures as well as strict infection control protocols-presurgical COVID-19 testing in high-risk cases, COVID-19 screening optimization, visitor restrictions, and appropriate disinfection between patients_-are in place.

The Impact of Postponing Treatment on Patients Although studies have focused on the effects of the COVID-19 pandemic on physicians practicing MMS, ${ }^{10}$

From the Department of Dermatology, University of New Mexico School of Medicine, Albuquerque.

The authors report no conflict of interest.

Correspondence: Naiara S. Barbosa, MD, Department of Dermatology, University of New Mexico School of Medicine, 1021 Medical Arts Ave NE, Albuquerque, NM 87102 (nbarbosa@salud.unm.edu).

doi: $10.12788 /$ cutis.0253 
little is known about the effects of delays in skin cancer treatment on patients. A survey conducted in the United Kingdom investigating the patient's perspective found that patients expressed worry and concern about the possibility that their MMS would be postponed and greatly appreciated continuation of treatment during the pandemic. ${ }^{11}$

Other medical specialties have reported their patient experiences during the pandemic. In a study examining patient perception of postponed surgical treatment of pelvic floor disorders due to COVID-19, nearly half of survey respondents were unhappy with the delay in receiving care. Furthermore, patients who reported being unhappy were more likely to report feelings of isolation and anxiety because their surgery was postponed. ${ }^{12}$ In another study involving patients with lung cancer, 9.1\% $(\mathrm{N}=15)$ of patients postponed their treatment during the COVID-19 pandemic because of pandemicrelated anxiety. ${ }^{13}$

With the goal of improving care at our institution, we conducted a brief institutional review board-approved survey to evaluate how postponing MMS treatment due to the COVID-19 pandemic affected patients. All MMS patients undergoing surgery in June 2020 and July $2020(N=99)$ were asked to complete our voluntary and anonymous 23-question survey in person during their procedure. We obtained 88 responses (response rate, $89 \%)$. Twenty percent of surveyed patients $(n=18)$ reported that their MMS had been postponed; $78 \%$ of those whose MMS was postponed $(n=14)$ indicated some level of anxiety during the waiting period. It was unclear which patients had their treatment postponed based on national guidelines and which ones elected to postpone surgery.

\section{Tips for Health Care Providers}

Patient-provider communication highlighting specific skin cancer risk and the risk vs benefit of postponing treatment might reduce anxiety and stress during the waiting period. ${ }^{14}$ A study found that COVID-19 posed a bigger threat than most noninvasive skin cancers; therefore, the authors of that study concluded that treatment for most skin cancers could be safely postponed. ${ }^{15}$ Specifically, those authors recommended prioritizing treatment for Merkel cell carcinoma, invasive SCC, and melanoma with positive margins or macroscopic residual disease. They proposed that all other skin cancers, including basal cell carcinoma, SCC in situ, and melanoma with negative margins and no macroscopic residual disease, could be safely delayed for as long as 3 months. ${ }^{15}$

For patients with multiple risk factors for COVID-19-related morbidity or mortality, delaying skin cancer treatment likely has less risk than contracting the virus. ${ }^{15}$ This information should be communicated with patients. Investigation of specific patient concerns is warranted, and case-by-case evaluation of patients' risk factors and skin cancer risk should be considered.
Based on the current, though limited, literature, delaying medical treatment can have a negative impact on the patient experience. Furthermore, proper precautions have been shown to limit intraoperative transmission of SARS-CoV-2 during MMS, but research is lacking. Practitioners should utilize shared decision-making and evaluate a given patient's risk factors and concerns when deciding whether to postpone treatment. We encourage other institutions to evaluate the effects that delaying MMS has had on their patients, as further studies would improve understanding of patients' experiences during a pandemic and potentially influence future dermatology guidelines.

\section{REFERENCES}

1. Center for Disease Control and Prevention. COVID-19. Accessed April 20, 2021. https://www.cdc.gov/coronavirus/2019-ncov/index.html

2. American College of Mohs Surgery. Mohs surgery ambulatory protocol during COVID pandemic (version 6-3-20). June 4, 2020. Accessed April 20, 2021. http://staging.mohscollege.org/UserFiles/AM20/Member\%20Alert /MohsSurgeryAmbulatoryProtocolDuringCOVIDPandemicFinal.pdf

3. COVID-19 resources. National Comprehensive Cancer Network website. Accessed April 20, 2021. https://www.nccn.org/covid-19

4. Narla S, Alam M, Ozog DM, et al. American Society of Dermatologic Surgery Association (ASDSA) and American Society for Laser Medicine \& Surgery (ASLMS) guidance for cosmetic dermatology practices during COVID-19. Updated January 11, 2021. Accessed April 10, 2021. https://www.asds.net/Portals/0/PDF/asdsa lasdsa-aslms-cosmetic-reopening-guidance.pdf

5. Geskin LJ, Trager MH, Aasi SZ, et al. Perspectives on the recommendations for skin cancer management during the COVID-19 pandemic. J Am Acad Dermatol. 2020;83:295-296. doi:10.1016/j.jaad.2020.05.002

6. Yuan JT, Jiang SIB. Urgent safety considerations for dermatologic surgeons in the COVID-19 pandemic. Dermatol Online J. 2020;26:1. Accessed April 20, 2021. http://escholarship.org/uc/item/2qr3w771

7. Otolaryngologists may contract COVID-19 during surgery. ENTtoday. March 20, 2020. Accessed April 20, 2021. https://www.enttoday.org /article/otolaryngologists-may-contract-covid-19-during-surgery/

8. Howard BE. High-risk aerosol-generating procedures in COVID-19: respiratory protective equipment considerations. Otolaryngol Head Neck Surg. 2020;163:98-103. doi:10.1177/0194599820927335

9. Tee MW, Stewart C, Aliessa S, et al. Dermatological surgery during the COVID-19 pandemic: experience of a large academic center. J Am Acad Dermatol. 2021;84:1094-1096. doi:10.1016/j.jaad.2020.12.003

10. Hooper J, Feng H. The impact of COVID-19 on micrographic surgery and dermatologic oncology fellows. Dermatol Surg. 2020;46:1762-1763. doi:10.1097/DSS.0000000000002766

11. Nicholson P, Ali FR, Patalay R, et al. Patient perceptions of Mohs micrographic surgery during the COVID-19 pandemic and lessons for the next outbreak. Clin Exp Dermatol. 2021;46:179-180. doi:10.1111/ced.14423

12. Mou T, Brown O, Gillingham A, et al. Patients' perceptions on surgical care suspension for pelvic floor disorders during the COVID-19 pandemic. Female Pelvic Med Reconstr Surg. 2020;26:477-482. doi:10.1097/SPV.0000000000000918

13. Fujita K, Ito T, Saito Z, et al. Impact of COVID-19 pandemic on lung cancer treatment scheduling. Thorac Cancer. 2020;11:2983-2986. doi:10.1111/1759-7714.13615

14. Nikumb VB, Banerjee A, Kaur G, et al. Impact of doctor-patient communication on preoperative anxiety: study at industrial township, Pimpri, Pune. Ind Psychiatry J. 2009;18:19-21. doi:10.4103/0972-6748.57852

15. Baumann BC, MacArthur KM, Brewer JD, et al. Management of primary skin cancer during a pandemic: multidisciplinary recommendations. Cancer. 2020;126:3900-3906. doi:10.1002/cncr.32969 